\begin{tabular}{|c|c|}
\hline Title & Genetic-A Igorithm A ssisted Design of C-Band CROW-Miniaturized PCW Interleaver \\
\hline Author(s) & Rosa, Lorenzo; Saitoh, Kunimasa; Kakihara, Kuniaki; Koshiba, Masanori \\
\hline Citation & $\begin{array}{l}\text { Journal of Lightwave Technology, 27(14), 2678-2687 } \\
\text { https://doi.org/10.1109/JLT.2009.2014883 }\end{array}$ \\
\hline Issue Date & 2009-07-15 \\
\hline Doc URL & http:/hdl. handle.net/2115/38859 \\
\hline Rights & $\begin{array}{l}\text { C 2009 IEEE. Personal use of this material is permitted. However, permission to reprint/republish this material for } \\
\text { advertising or promotional purposes or for creating new collective works for resale or redistribution to servers or lists, } \\
\text { or to reuse any copyrighted component of this work in other works must be obtained from the IEEE. }\end{array}$ \\
\hline Type & article \\
\hline File Information & 27-14_p2678-2687.pdf \\
\hline
\end{tabular}

Instructions for use 


\title{
Genetic-Algorithm Assisted Design of C-Band CROW-Miniaturized PCW Interleaver
}

\author{
Lorenzo Rosa, Member, IEEE, Kunimasa Saitoh, Member, IEEE, Kuniaki Kakihara, and \\ Masanori Koshiba, Fellow, IEEE
}

\begin{abstract}
We investigate the design of a miniaturized C-band photonic-crystal waveguide (PCW) interleaver, employing coupled-resonator optical waveguide (CROW) ring resonators. An optimization approach based on a genetic algorithm (GA) has been applied to the filter components, such as tapers and directional couplers, leading to low reflection and high phase linearity. To evaluate the interleaver transmittivity function, an S-matrix approach is worked out, based on the optimized building blocks. A sensitivity analysis is performed to evaluate the effect of parameter tolerances.
\end{abstract}

Index Terms-Finite-element methods, genetic algorithms, optical directional couplers, optical waveguide filters, scattering matrices.

\section{INTRODUCTION}

$\mathbf{I}$ $\mathrm{N}$ wavelength-division multiplexing (WDM) systems, wavelength selective filters are recognized as fundamental building blocks, while at the same time photonic crystal (PC) based waveguides represent a key technology in the effort to improve optical integrated circuits performance. Several works have considered filters for single-band operation, e.g., notch-rejection filters [1], [2] and channel-drop filters [3]-[6]. Interleave filters are considered key components in these systems for their ability to multiplex and demultiplex sets of periodically spaced wavelengths with narrow separation. An interleaver has a periodical transfer function, originally designed to separate or combine even channels and odd channels across a dense WDM (DWDM) comb [7]. Required characteristics are a minimum insertion loss and rapid roll-off on the band edges. Moreover, a flat-top passband is considered an important requirement for several kinds of optical filters [8], [9].

Interleavers can be realized with optical delay-line circuits employing directional couplers and Mach-Zehnder interferometers (MZIs) [10]-[13], while the filter architecture can be designed in either of two categories, the simplest one being a finite-impulse response (FIR) configuration based on delay lines and directional couplers [7], to which feedback loops such as

Manuscript received September 22, 2008; revised January 28, 2009. First published April 24, 2009; current version published July 01, 2009. This work was supported in part by the Global COE Program at Hokkaido University and by the JSPS Research Fellowship for Young Scientists.

The authors are with the Division of Media and Network Technologies, Graduate School of Information Science and Technology, Hokkaido University, Sapporo 060-0814, Japan (e-mail: 1rosa@ieee.org; ksaitoh@ist. hokudai.ac.jp; kakihara@icp.ist.hokudai.ac.jp; koshiba@ist.hokudai.ac.jp).

Color versions of one or more of the figures in this paper are available online at http://ieeexplore.ieee.org.

Digital Object Identifier 10.1109/JLT.2009.2014883 ring resonators can be added to obtain an infinite-impulse response (IIR) configuration [14]. The interest of this work is the design of a miniaturized interleaver with flat-top passbands, for integration in PC circuits. It is known that the flat-top spectral response depends on the filter order, and the IIR configuration has the advantage of attaining flat-top passband and rapid roll-off with a low number of resonators, so in this implementation, the interleaver is realized as a photonic-crystal waveguide (PCW) fifth-order IIR elliptic half-band filter [14], employing two 3-dB couplers and a MZI whose arms are loaded by hexagonal ring resonators, as shown in Fig. 1(a). The PC lattice is constructed with Si rods having pitch $a=0.45 \mu \mathrm{m}$ and radius $r=0.25 a$, embedded in a $\mathrm{SiO}_{2}$ substrate. In order to miniaturize photonic structures, designs employing coupled-resonator optical waveguides (CROWs) have been proposed, e.g., for reducing the MZI size, albeit with reduced flatness [15]. In this work, CROWs with low group velocity [16] are used to increase the effective length of the ring resonator structures and of the MZI arms, to reduce the overall size while preserving as much as possible the passband flatness of the device. As shown in Fig. 1(a), the CROW is constructed by inserting Si rods of radius $r_{c}$ inside the PCW, creating a sequence of cavities. Auxiliary rods of radius $r_{s}$ are inserted inside the cavities to tune the CROW dispersion characteristics and improve matching when connecting to PCW sections, where they are present throughout. Fig. 1(b) shows the dispersion curves of the rod-loaded PCW, and the CROW when the radii $r_{c}=0.18 a$ and $r_{s}=0.07 a$ are used. The interleaver also includes phasing sections named $\varphi, \varphi_{11}$ and $\varphi_{12}$, and each of them is designed by connecting two CROW subsections with the radii $r_{c}$ and $r_{s}$ modified to equalize the phase variations of the building blocks in the MZI long arm and in the rings, maintaining the correct phase relationships throughout C-band.

The complexity of the structure prevents a direct full-wave simulation, so it is divided in its primitive building blocks, which are then separately optimized. The transmittance of the whole structure is then derived from calculations of their transfer functions. The necessity to maintain a high transmittivity and a flat-top passband over the whole C-band requires accurate control of the dispersion characteristics of the building blocks, obtained through modification of a large number of rod radii. This requires the adoption of an optimization approach based on the introduction of a genetic algorithm (GA).

To achieve the correct functioning of the MZI on which the filter is based, as depicted in Fig. 1(a), the coupling between ring and arm must be held at a constant level throughout C-band. Two kinds of couplers are used in the MZI: a 60/40 coupler (1.5:1 power ratio) for the long arm and a 97/03 coupler (33.5:1 ratio) for the short arm, whose coupling ratios are fixed by the filter 


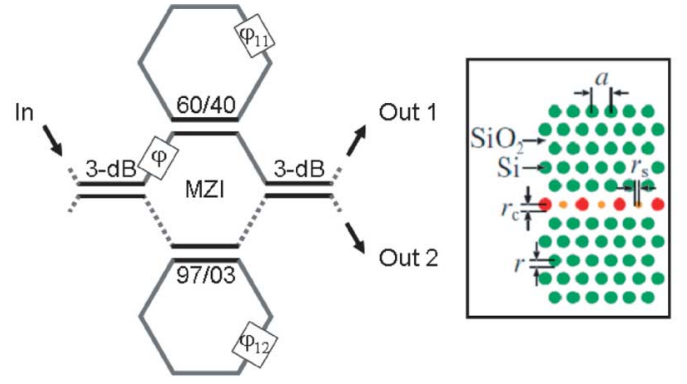

(a)

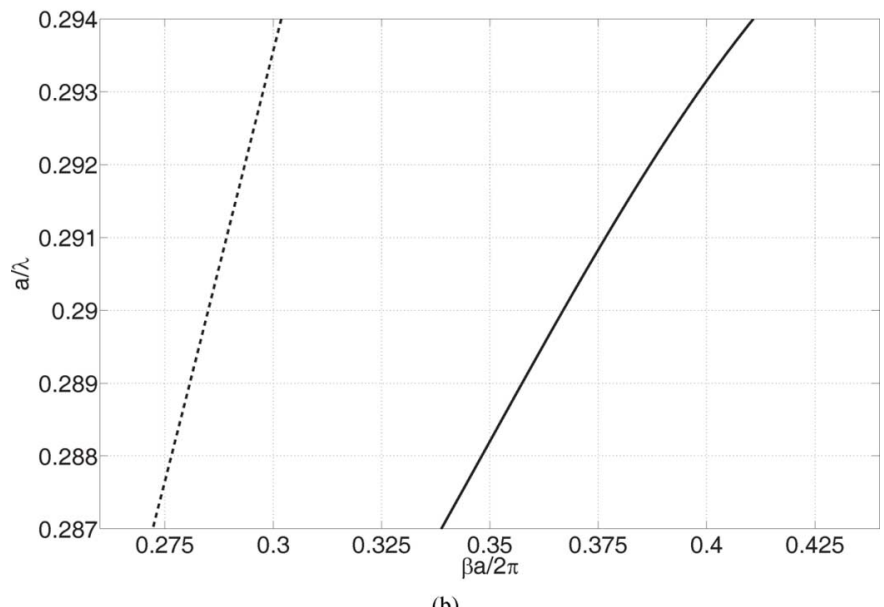

(b)

Fig. 1. (a) Interleaver layout ( $a=0.45 \mu \mathrm{m}, r=0.25 a$, CROW structure on the right), showing PCW (dashed) and CROW (solid) sections. (b) Dispersion curves on C-band for CROW (solid, $r_{c}=0.18 a, r_{s}=0.07 a$ ) and PCW (dashed, $r_{c}=r_{s}=0.07 a$ ).

parameters. The key to obtaining broadband coupling is the establishment of even and odd coupler supermodes with parallel dispersion curves throughout the band [17]; however, a careful optimization of the Si rod diameters in the vicinity of the transition with the input/output PCW is required for also keeping a broadband power matching.

The transfer function of the interleaver structure can be worked out, based on the S-matrices of the building blocks (PCW, CROW, matching tapers, bends, phasing sections, couplers). These are calculated from the complex amplitudes of the reflected and transmitted waves, obtained through 2-D finite-element method (FEM) simulations [18]. Symmetry conditions of the single devices can be exploited to reduce the number of matrix terms to be calculated.

The device structure models used in the simulations are depicted in Fig. 2. The taper structure shown in Fig. 2(a) is employed to reduce reflection at the transition between PCW and CROW sections, and the dotted circles in the figure emphasize the $10 \mathrm{Si}$ rods whose radii $r_{1}, \ldots, r_{10}$ are optimized by the GA. In the case of the CROW bend structure shown in Fig. 2(b), six rods surrounding the bend are optimized, having four different radii $r_{1}, \ldots, r_{4}$ for symmetry reasons. The model for the coupler structure in Fig. 2(c) is more complex, owing to the necessity to optimize 40 rods with 10 different radii $r_{1}, \ldots, r_{10}$ near the coupling interfaces with the PCW, in the case of the 97/03 coupler. For the $3-\mathrm{dB}$ and $60 / 40$ couplers, seven rod radii are optimized, setting $r_{8}=r_{9}=r$ and $r_{10}=r_{s}$. The rods in the

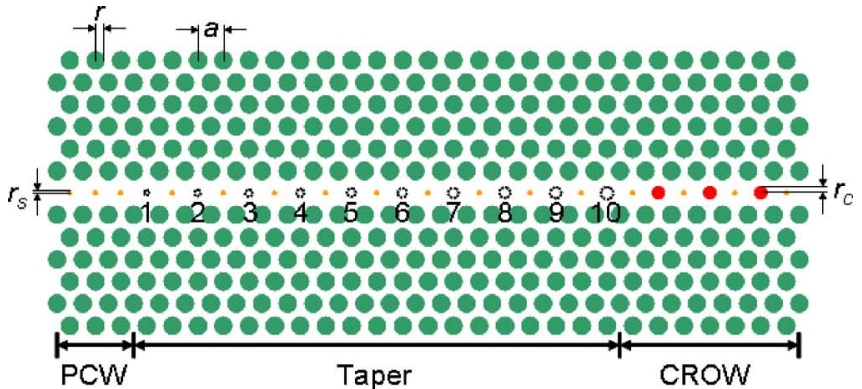

(a)

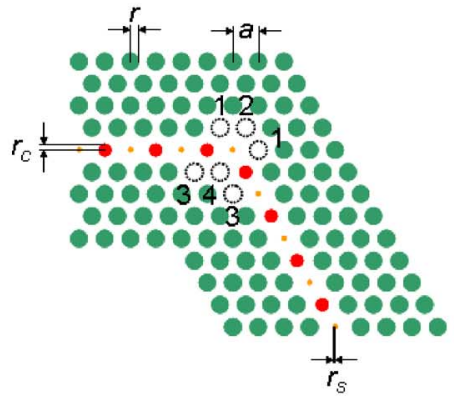

(b)

Port 1

Port 2

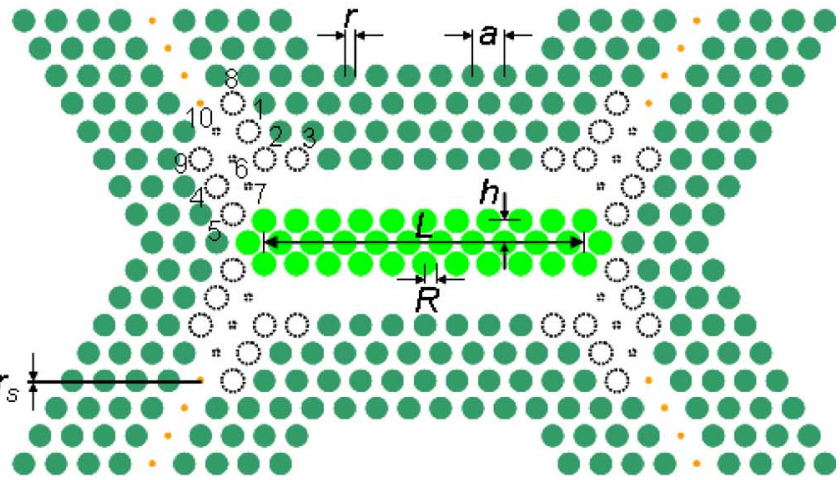

Port 3

Port 4

(c)

Fig. 2. Structure models of the (a) PCW to CROW taper, (b) $60^{\circ}$ CROW bend, and (c) PCW directional couplers $(h=0.433 a, R=0.275 a)$. The numbered dotted circles mark the GA-optimized rods, in the directional coupler case only port 1 is shown, the others are numbered symmetrically.

coupling region have radius $R=0.275 a$, while the distance between the rows is $h=0.433 a$. The coupling length $L$ is equal to $10 a$ for the $3-\mathrm{dB}$ coupler, $11 a$ for the $60 / 40$ one, and $22 a$ for the $97 / 03$ one. In the discussion, port 1 of the couplers is designated as the input port.

The full transmittivity and reflectivity of the interleaver are calculated through a recursive approach, based on the S-matrices of the 3-dB coupler and of the MZI arms. The optical signals are propagated alternatively in the forward and backward directions, updating the complex amplitudes of the incident and reflected waves at the device ports, until convergence is obtained. Plots are given of transmittivity of the two output ports and reflectivity back to the two input ports, in the hypothesis of absence of waveguide losses and for a first-order estimate of them. 


\section{GENETIC ALGORITHM}

The GA is an optimization method whose specifications stem from the rules of natural selection, which is the process that drives biological evolution [19]. The method works by establishing a population of individual solutions, whose "genes" represent the problem parameters, and repeatedly modifying it in successive generations. At each step, the algorithm selects individuals from the current population to be parents and uses them to produce the children for the next generation. The parents are chosen through a random process weighted by the evaluation of their "fitness" as solution candidates, which is called selection. This is done on the basis of the value of a function called "fitness function" or "objective function," which is computed for every individual in the population. Over successive generations, the population "evolves" toward an optimal solution.

The GA can be applied to solve a variety of optimization problems that are not well suited for standard optimization algorithms, such as problems which can be expressed as the minimization of a certain function, when the function has many local minima which make it difficult for standard, gradient-based methods to find the global minimum. Also, it is very useful for optimizing functions depending on a large number of mutually interacting parameters, which precludes effective manual optimization. The method can also work to obtain solutions bounded by constraints of physical or mathematical nature.

The algorithm begins by creating a random initial population, then steps through a sequence of new generations created from the individuals in the current generation, by cycling through the following.

1) Each member of the current population is evaluated by computing its fitness value.

2) The raw fitness scores are scaled to convert them into a more usable range of values.

3) Parents are selected, based on their fitness.

4) Some of the individuals having the best fitness are chosen as elite individuals and directly passed to the next population.

5) Children are produced from the parents, either by making random changes to a single parent (mutation) or by combining the gene entries of a pair of parents (crossover).

6) The current population is replaced with the children to form the next generation.

The algorithm keeps cycling until it meets the stopping criteria, defined by the user and devised to end the optimization process when the result is deemed satisfactory. The "chromosome" defining the individuals is implemented as a vector, whose gene entries represent the radii of the $\mathrm{Si}$ rods to be optimized.

After scoring, the two best individuals are selected as elite individuals and automatically passed on to the next generation. Of the remaining children, a fraction $k$ is created by crossover and the remaining $1-k$ by mutation, with the crossover fraction $k$ initially set to 0.8 and subsequently optimized. The selection process is weighted by fitness, so that the best fit individuals are more likely to be selected as parents. To create crossover children, at each coordinate of the child vector, a gene is randomly selected at the same coordinate from one of the two parents. Mutation children are created by randomly changing the genes of individual parents: the algorithm adds a random vector from a Gaussian distribution to the parent vector.

The two-port taper and bend devices should be optimized for two objectives: high transmittivity and passband flatness. In the present implementation, the algorithm works by minimizing the fitness function, which has been chosen to fulfil both objectives, assigning the best score to the individual best approximating flat unity transmittivity. It computes the deviation from unity of the individual's transmittivity curve over C-band, by sampling the curve at 18 wavelengths between $1530 \mathrm{~nm}$ and $1565 \mathrm{~nm}$, calculating the absolute distance from unity for each wavelength and summing the squares of the distances. The transmittivity curve is sampled by running several single-wavelength finite-element frequency-domain (FEFD) simulations. The FEM mesh is automatically generated from the chromosome data. The fitness function is defined as

$$
\text { Fitness }=\sum_{\lambda_{i}}\left[1-T\left(\lambda_{i}\right)\right]^{2}
$$

where $T\left(\lambda_{i}\right)$ is the value of the transmittivity sampled at wavelength $\lambda_{i}$ with $i=1, \ldots, n$. This choice has the effect of amplifying differences at single wavelengths, so that single wavelengths or groups of wavelengths with high difference from unity will have a greater impact on fitness. This results in a higher fitness penalty for individuals that exhibit poor transmittivity flatness.

The GA optimization of the couplers is a multi-objective problem, as the transmittivity of the through port (port 2 in Fig. 2) and of the coupled port (port 4) must be optimized concurrently for a flat response equal to the respective coupling coefficient. Accordingly, power coupled to the isolated port (port 3) should be negligible. The fitness function used computes the deviation from coupling coefficient $C_{i}$ of the respective port transmittivity curve $T_{i}(\lambda)$ over C-band, as

$$
\text { Fitness }=\sum_{\lambda_{i}}\left[C_{2}-T_{2}\left(\lambda_{i}\right)\right]^{2}+\alpha \sum_{\lambda_{i}}\left[C_{4}-T_{4}\left(\lambda_{i}\right)\right]^{2}
$$

where $C_{4} / C_{2}$ is the coupling ratio and the $\alpha$ coefficient amplifies the coupled port fitness, more difficult to optimize for high coupling ratios. Satisfactory performance was obtained for $\alpha=1.0$ in the 60/40 coupler case, and for $\alpha=5.0$ in the $97 / 03$ coupler one.

In optimizing the phasing sections in the long arm of the MZI and in the ring resonators, a strategy has been devised for applying the GA optimization method. These sections are designed by varying the large-rod and small-rod radii in the CROW, so as to synthesize a dispersion curve with a value and slope of phase constant $\beta$ suitable for providing a constant phase shift and delay according to specifications. Moreover, given the large phase characteristics variation with wavelength in the CROW, the $\beta$ curve must be designed to equalize shift and delay on the whole $\mathrm{C}$-band, requiring the use of a two-stage phasing network.

In the long MZI arm, made of CROW with phase constant $\beta_{c}$, the path difference must be $\Delta L=v_{g} /(2 \Delta f)$, where $v_{g}$ is the CROW group velocity and $\Delta f$ is the DWDM comb channel spacing, with respect to the lower arm made of PCW with phase 
constant $\beta_{p}$, keeping a constant phase shift $\varphi=0$. The phase delay of the long arm is thus written as

$$
\varphi_{\text {long }}=\beta_{a 1} \Delta L_{a 1}+\beta_{a 2} \Delta L_{a 2}+\beta_{c} \Delta L_{c}+\varphi_{21, \text { lac }}+\varphi_{\text {tapers }}
$$

where $\beta_{a 1}$ and $\Delta L_{a 1}$ refer to the first phasing subsection, and $\beta_{a 2}$ and $\Delta L_{a 2}$ refer to the second one, while $\Delta L_{c}$ is the CROW length. $\varphi_{21 \text { lac }}$ is the phase shift introduced by the long-arm coupler on the propagation path from port 1 to port 2 , while $\varphi_{\text {tapers }}$ is the combined phase shift introduced by the tapers to the CROW and phasing sections. The phase delay of the short arm is written as

$$
\varphi_{\text {short }}=\beta_{p} \Delta L_{p}+\varphi_{21, \mathrm{sac}}
$$

where $\Delta L_{p}$ is the PCW length and $\varphi_{21 \text {,sac }}$ is the phase shift introduced by the short-arm coupler on the propagation path from port 1 to port 2 .

In this case, the fitness function is written as

$$
\text { Fitness }=\sum_{\lambda_{i}}\left[\varphi_{\text {long }}\left(\lambda_{i}\right)-\varphi_{\text {short }}\left(\lambda_{i}\right)-\beta_{c} \Delta L\right]^{2}
$$

which is minimized when the path difference condition is fulfilled, being $\varphi_{[\text {long,short }]}\left(\lambda_{i}\right)$ the respective phase sampled at wavelength $\lambda_{i}$ with $i=1, \ldots, n$.

The phasing section in each ring must provide a constant path difference $2 \Delta L$, while keeping a constant phase shift $\varphi_{11}=$ $\varphi_{12}=\pi$. In this case the phase delay of the long-arm and short-arm ring is expressed as

$$
\begin{array}{r}
\varphi_{\text {ring, }[l, s]}=\left[\beta_{r 1} \Delta L_{r 1}+\beta_{r 2} \Delta L_{r 2}+\beta_{c} \Delta L_{c}+\varphi_{\text {dev }}\right]_{[l, s]} \\
+\varphi_{21,[\text { lac }, \text { sac }]}
\end{array}
$$

where $\beta_{r 1}$ and $\Delta L_{r 1}$ refer to the first phasing subsection, $\beta_{r 2}$ and $\Delta L_{r 2}$ refer to the second one, while $\Delta L_{c}$ is the CROW length. $\varphi_{21,[\mathrm{lac}, \mathrm{sac}]}$ is the phase shift introduced by the respective ring coupler, while $\varphi_{\mathrm{dev}}$ is the combined phase shift introduced by the connecting devices between the elements that make up the ring.

In this case, the fitness function is

$$
\operatorname{Fitness}_{[l, s]}=\sum_{\lambda_{i}}\left[\varphi_{\text {ring, }[l, s]}\left(\lambda_{i}\right)-\beta_{c} 2 \Delta L-\pi\right]^{2}
$$

which is also minimized when the path difference condition is fulfilled, being $\varphi_{\text {ring, }[l, s]}\left(\lambda_{i}\right)$ the respective ring phase sampled at wavelength $\lambda_{i}$ with $i=1, \ldots, n$.

The phasing sections are evaluated for phase flatness over the band by the GA, using a chromosome with six genes, corresponding to the values of $r_{c}, r_{s}$, and $\Delta L$ of each of the two CROW subsections forming each phasing section. The length of the remaining spans of waveguide (CROW for the rings and upper arm of the MZI, PCW for the lower arm) is computed so that the phase equalization will be optimal near the center of the band.

\section{TRANSFER FunCtion}

The present implementation is aimed for a flat-top passband in C-band with minimum insertion loss and rapid roll-off at the band edges, building a PCW structure which realizes a fifth-order IIR elliptic half-band filter [14]. The design choice is due to the elliptic filter having the fastest roll-off at the band edges, important for narrowly-spaced combs. Moreover, the interleaver periodical transfer function has equally wide passbands and stop-bands, implicitly respecting the half-band power law, which enables the use of a half-band filter, featuring the same performance at reduced complexity.

The transmittance of the filter can be derived in closed form as [20]

$$
\begin{aligned}
T & =-j\left\{F_{11} \cos \theta_{1} \sin \theta_{2} e^{-j \beta \Delta L}+F_{12} \sin \theta_{1} \cos \theta_{2}\right\} \\
F_{i j} & =\frac{\cos \theta_{i j}-e^{-j\left(2 \beta \Delta L+\varphi_{i j}\right)}}{1-\cos \theta_{i j} e^{-j\left(2 \beta \Delta L+\varphi_{i j}\right)}}
\end{aligned}
$$

where the $\theta_{i}$ and $\theta_{i j}$ represent the coupling coefficients of the directional couplers $\left(\theta_{1}\right.$ and $\theta_{2}$ are related to the $3-\mathrm{dB}$ couplers, $\theta_{11}$ to the $60 / 40$ one and $\theta_{12}$ to the $97 / 03$ one), while the $\varphi_{i j}$ are the phase shifts introduced by the phasing sections and $\beta$ is the phase constant of the employed waveguide.

This approximation is only valid in the hypothesis of ideal waveguides and couplers, and absence of reflection, so a more accurate method is envisioned that would use the complex amplitudes of reflection and transmission returned by the FEM solver in order to build the S-matrices related to the building blocks. The optical circuit structure would then give the network topology employed to set up a system of matrix equations. To simplify the calculations, an analytical solution is worked out for the S-parameters of the feed-back loops formed by the two ring-coupler structures.

The ring-coupler is divided in two components, being the directional coupler and the ring. The incident wave on coupler port $i$ [according to Fig. 2(c)] is named $a_{i}$, and the reflected one $b_{i}$, while the incident and reflected waves on the ring ends are named $a_{r i}$ and $b_{r i}$, respectively. Based on this definition and on the symmetries of the structures, the S-matrices are constructed, named $S$ for the coupler and $S_{r}$ for the ring. $S$ is a $4 \times 4$ matrix and $S_{r}$ a $2 \times 2$ one, while the connection between the two devices also sets a number of boundary conditions, namely $a_{4}=b_{r 1}, b_{4}=a_{r 1}, a_{3}=b_{r 2}$, and $b_{3}=a_{r 2}$.

The problem under scrutiny involves exploiting the boundary conditions so as to write $a_{3}$ and $a_{4}$ as a function of $a_{1}$ and $a_{2}$, thus reducing the combined structure to a two-port device. To achieve this, firstly the boundary conditions are plugged into the equations obtained from matrix $S_{r}$, writing

$$
\left\{\begin{array}{l}
a_{3}=b_{4} s_{r 21}+b_{3} s_{r 11} \\
a_{4}=b_{4} s_{r 11}+b_{3} s_{r 21}
\end{array}\right.
$$

which are in turn plugged into the equations for $b_{3}$ and $b_{4}$ obtained from the last two rows of the $S$ matrix. These are then plugged back into (10), where, for the sake of this discussion, five coefficients are defined and named $c, d, e, f$ and $g$, as follows:

$$
\left\{\begin{array}{l}
c=s_{11} s_{r 11}+s_{21} s_{r 21} \\
d=s_{11} s_{r 21}+s_{21} s_{r 11} \\
e=\frac{1-c}{(1-c)^{2}-d^{2}}
\end{array}\right.
$$




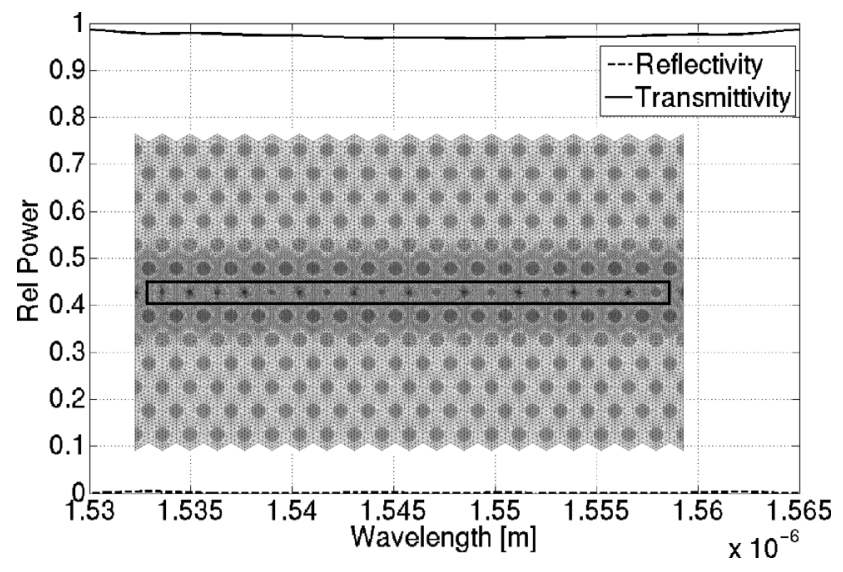

Fig. 3. Transmittivity and reflectivity curves on C-band for GA-optimized 10-periods PCW to CROW taper (optimal structure in inset). The black box highlights the region containing the optimized rods.

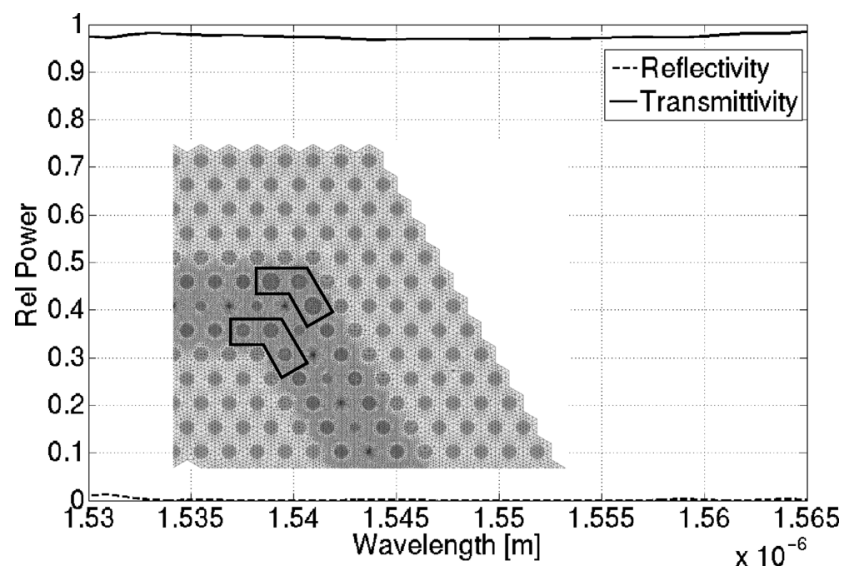

Fig. 4. Transmittivity and reflectivity curves on C-band for GA-optimized CROW bend (optimal structure in inset). The black boxes highlight the optimized rods.

$$
\left\{\begin{array}{l}
f=\left(s_{41}+s_{31} \frac{d}{1-c}\right) s_{r 21}+\left(s_{31}+s_{41} \frac{d}{1-c}\right) s_{r 11} \\
g=\left(s_{31}+s_{41} \frac{d}{1-c}\right) s_{r 21}+\left(s_{41}+s_{31} \frac{d}{1-c}\right) s_{r 11}
\end{array}\right.
$$

so that the result can be written as

$$
\left\{\begin{array}{l}
a_{3}=e f a_{1}+e g a_{2} \\
a_{4}=e g a_{1}+e f a_{2}
\end{array} .\right.
$$

Finally, (13) are plugged into the equations for $b_{1}$ and $b_{2}$ obtained from the first two rows of the $S$ matrix. The final equations describing the ring-coupler device are thus obtained as follows:

$$
\left\{\begin{array}{l}
b_{1}=\left(s_{11}+e f s_{31}+e g s_{41}\right) a_{1}+\left(s_{21}+e g s_{31}+e f s_{41}\right) a_{2} \\
b_{2}=\left(s_{21}+e f s_{41}+e g s_{31}\right) a_{1}+\left(s_{11}+e g s_{41}+e f s_{31}\right) a_{2}
\end{array} .\right.
$$

Once the building blocks are optimized and their S-matrices are known, it is possible to calculate the full transfer function through matrix algebra by combining the matrix $S_{c}$ of the 50/50 coupler with the matrix $S_{m}$ of the MZI. They can both be simplified by exploiting device symmetries and by noticing that there is no cross-coupling between MZI ports at opposite corners. The approach presented in [21] is considered and extended

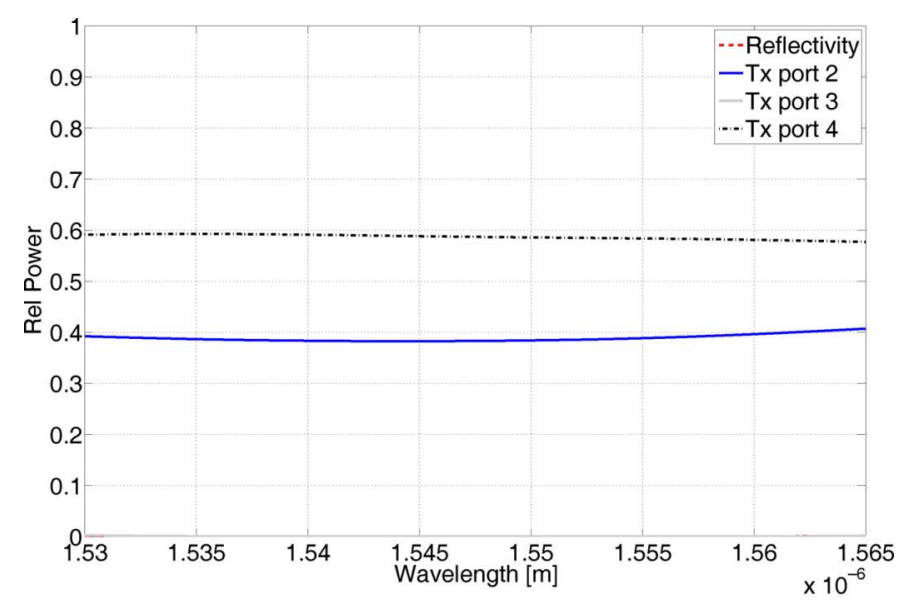

(a)

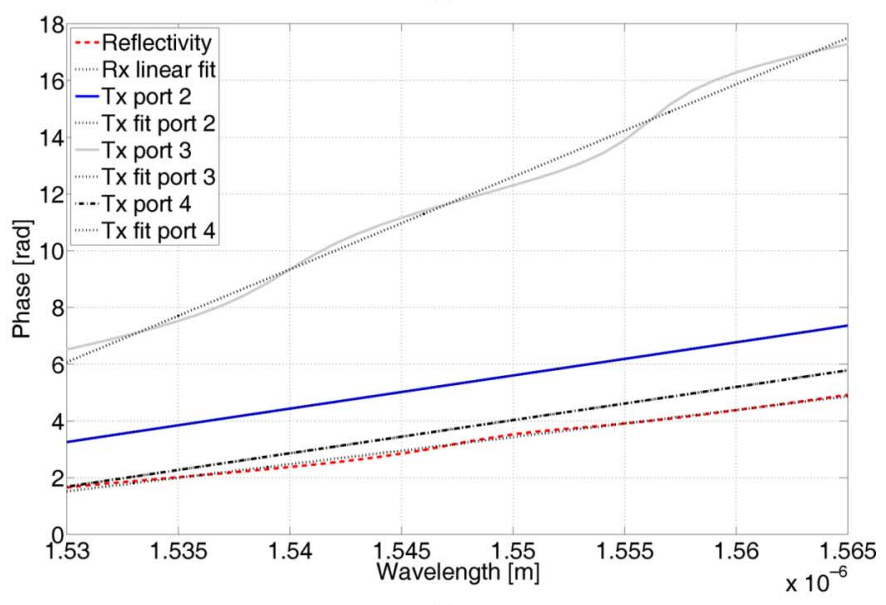

(b)

Fig. 5. (a) Transmission and (b) phase curves on C-band for GA-optimized 60/40 coupler.

by employing complex S-coefficients derived from the amplitude-phase results of the full-wave FEM simulations.

The device thus is characterized by four interfaces, being the input interface, the one between first coupler and MZI, the one between MZI and second coupler, and the output interface. The transmittivity and reflectivity are obtained through a recursive calculation which proceeds by successively updating the forward propagating waves up to the output interface. Then it successively updates the backwards propagating waves up to the input interface, cycling until the solutions at the input and output interfaces converge to stable values.

\section{NUMERICAL RESULTS}

First, the GA optimization has been applied to a taper structure, necessary to couple the $\mathrm{CROW}$ which composes the ring resonators and the upper arm of the MZI to the PCW which composes the remainder of the device. To reduce mismatch, small-diameter Si rods $\left(r_{s}=0.07 a\right.$ in Fig. 1) are located in the PCW and inside the CROW cavities. The proposed taper has a length of ten CROW periods, and the simulation domain comprises two such tapers placed back-to-back, one of which is shown in the inset of Fig. 3 .

The chromosome describing the individual is a vector with ten entries corresponding to the progressively varying radii of 


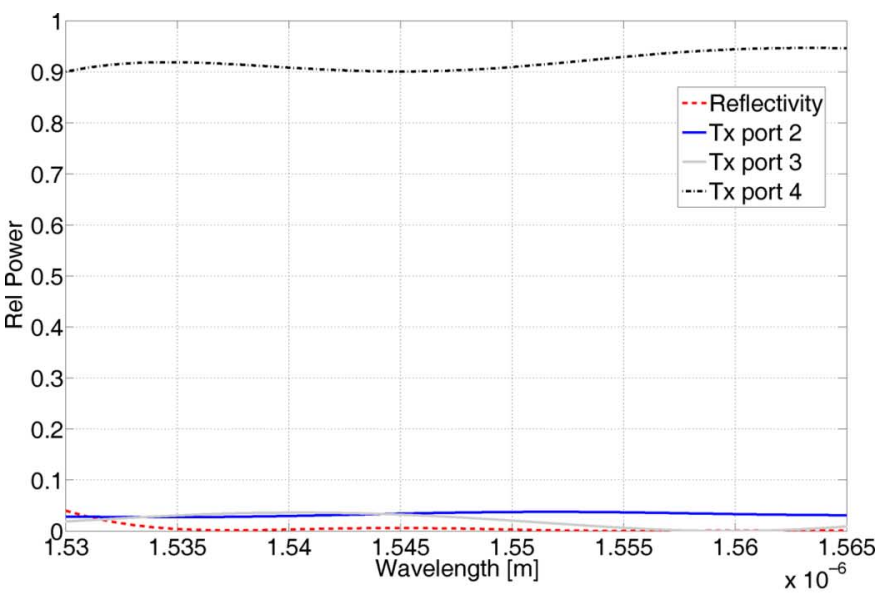

(a)

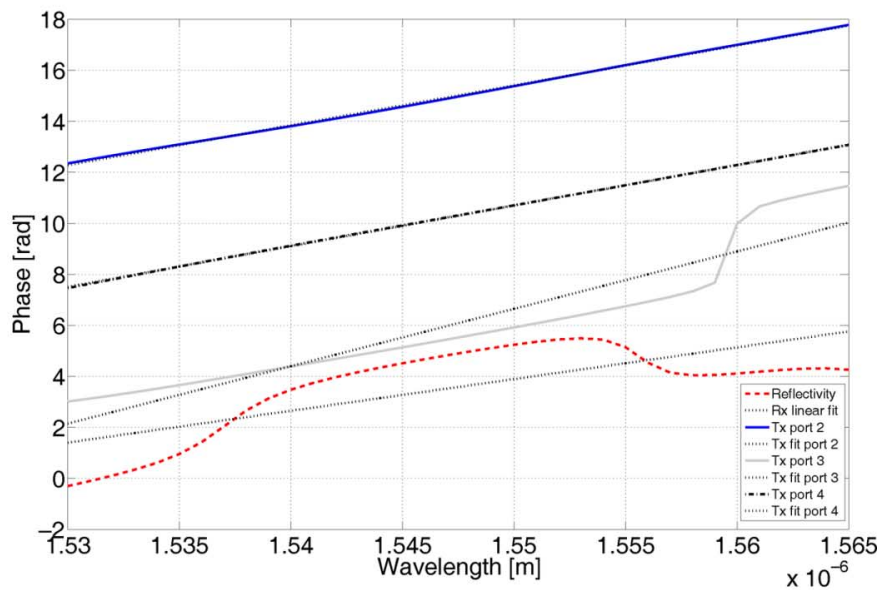

(b)

Fig. 6. (a) Transmission and (b) phase curves on C-band for GA-optimized $97 / 03$ coupler.

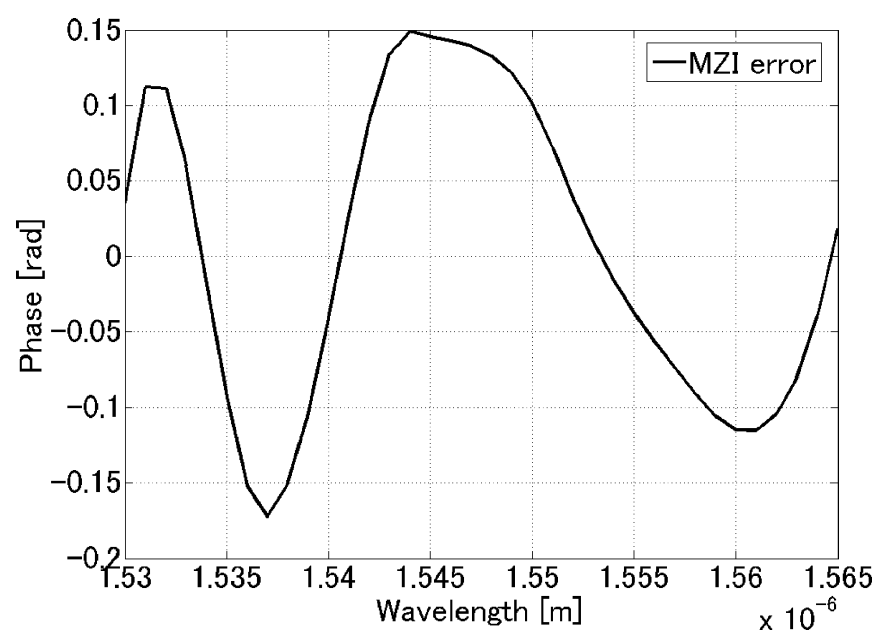

Fig. 7. Approximation error of GA-optimized MZI phasing section.

the Si taper rods. As initial condition, the genetic algorithm is fed with radii values which vary linearly from $r_{s}$ to the CROW rod radius $\left(r_{c}=0.18 a\right.$ in Fig. 1).

$\mathrm{xNext}$, the GA has been applied to a $60^{\circ} \mathrm{CROW}$ bend, which is necessary to build the ring resonator. For coupling purposes, the simulated structure implements a bend connected to the PCW by two tapers corresponding to the optimum individual found in the previous step. The optimized bend is shown in

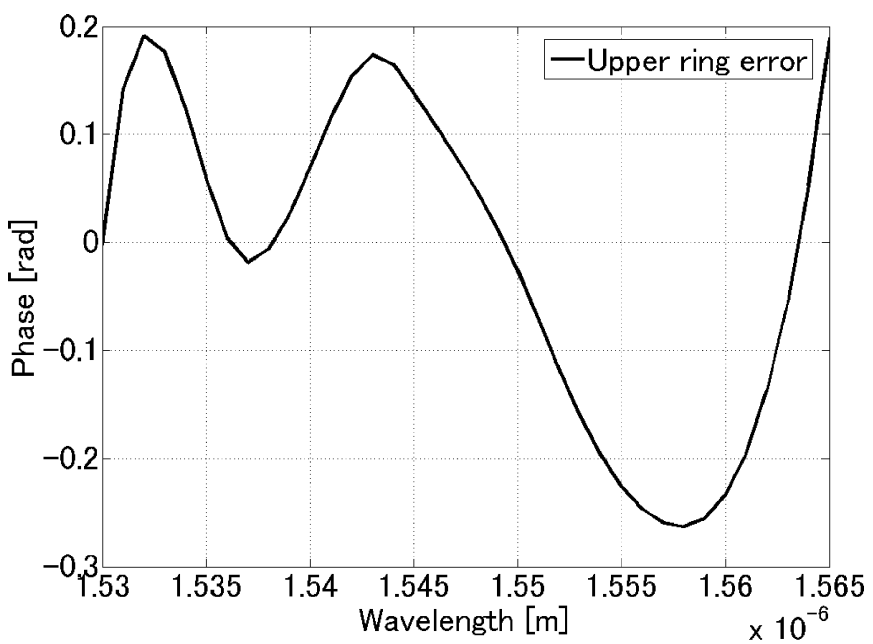

(a)

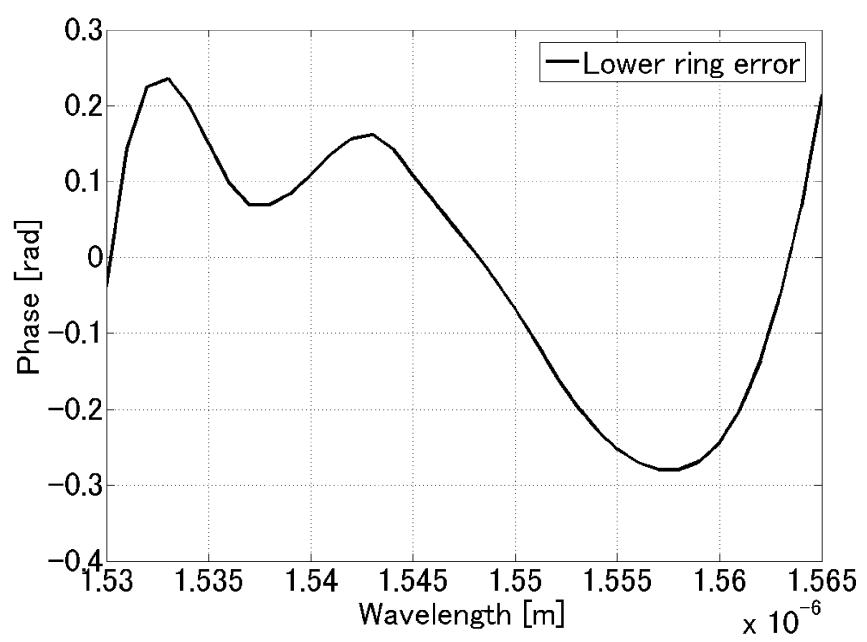

(b)

Fig. 8. Approximation error of GA-optimized (a) upper and (b) lower ring phasing sections.

the inset of Fig. 4. The optimization is applied to the six rods surrounding the bend, whose radii for symmetry reasons can be described with a 4-entry chromosome. As initial condition, the radii are set equal to the rods in the surrounding photonic crystal.

In all cases, the algorithm has been run for 50 generations, producing transmission spectra of $97 \%$ or higher over the whole C-band, as shown in Figs. 3 and 4. Even more remarkable is the flatness obtained, which has a very good $1.5 \%$ value on the band. In the optimum structure obtained for the taper, the radii $r_{1}, \ldots, r_{10}$ are equal to $0.0829 a, 0.0811 a, 0.09611 a$, $0.1107 a, 0.12342 a, 0.13687 a, 0.14448 a, 0.15397 a, 0.16739 a$, and $0.17813 a$, respectively. In the optimum bend case, the radii $r_{1}, \ldots, r_{4}$ are equal to $0.31479 a, 0.27569 a, 0.2437 a$, and $0.22837 a$, respectively.

The obtained result for the 60/40 coupler is shown in Fig. 5. The optimization is applied to the seven rods surrounding the PCW transition, and the algorithm is run for 50 generations on a population of 21 individuals, obtaining a mean coupling ratio of $1.51: 1$ on the whole band, with a $8 \%$ overall flatness and very good decoupling of the isolated port, as shown in Fig. 5(a). 


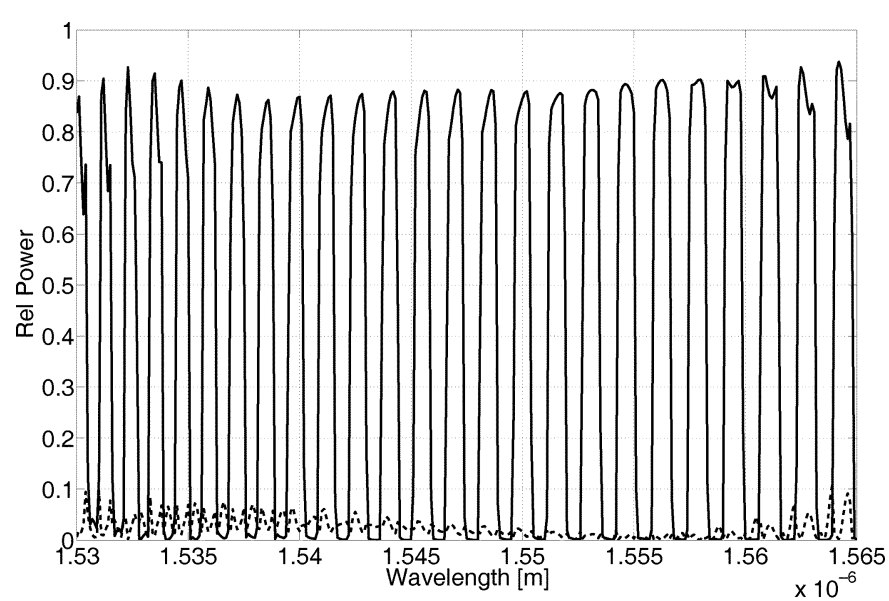

(a)

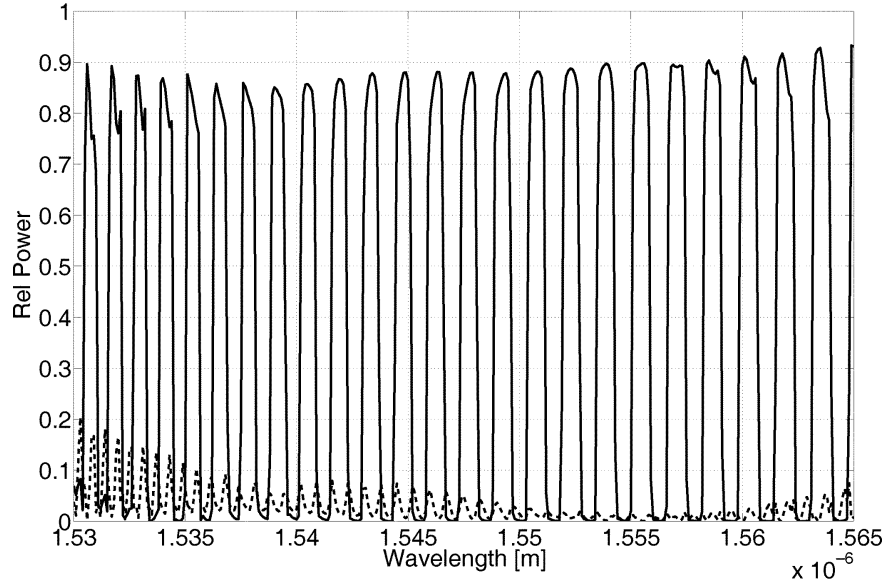

(b)

Fig. 9. Transmittivity (solid) and reflectivity (dashed) for the (a) upper side and (b) lower side of the interleaver. No loss is introduced for the waveguide.

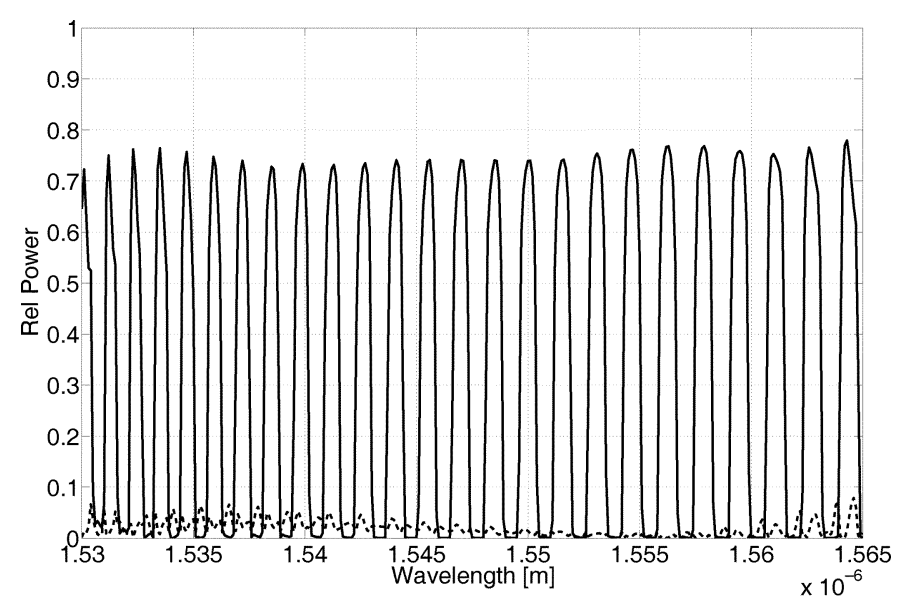

(a)

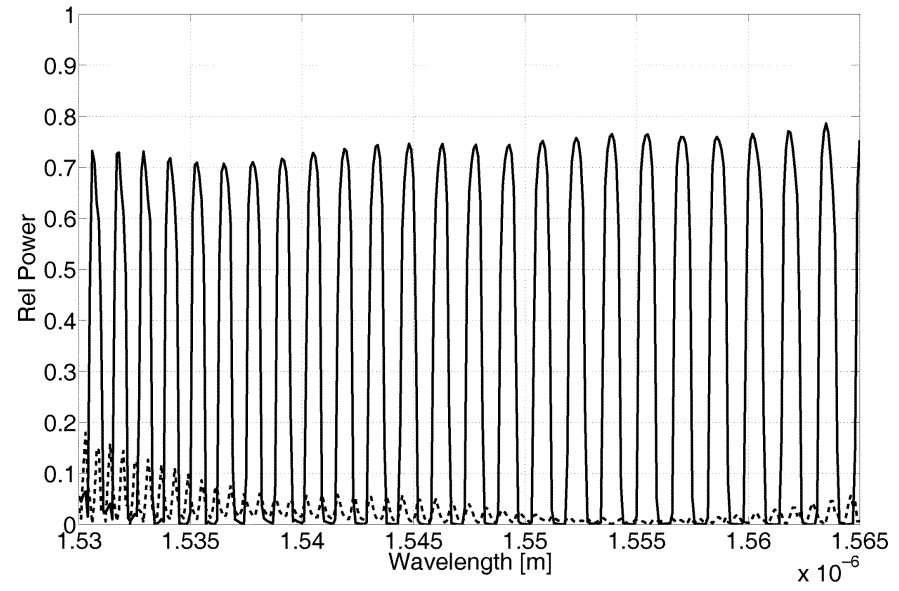

(b)

Fig. 10. Transmittivity (solid) and reflectivity (dashed) for the (a) upper side and (b) lower side of the interleaver. Estimated waveguide losses are introduced in the model.
The phase response has been computed and shown in Fig. 5(b), where from the comparison with the linear curve fit, it can be noticed that the transfer function for the through and coupled ports has very high linearity.

In the case of the 97/03 coupler, the GA is run for 50 generations on a 10-rod chromosome structure, producing the individual whose transmittivity curves are shown in Fig. 6(a). The mean coupling ratio is $28.72: 1$, with an overall $33 \%$ flatness. The change in ratio over the band is mostly due to reflection and partial coupling to the isolated port, however the phase response for the through and coupled ports shown in Fig. 6(b) is highly linear, making it easier to optimize the phasing sections.

The optimum structure obtained for the 97/03 coupler has radii $r_{1}, \ldots, r_{10}$ equal to $0.23755 a, 0.23796 a, 0.32502 a$, $0.24287 a, \quad 0.16674 a, 0.075435 a, 0.0974 a, 0.22591 a$, $0.31877 a$, and $0.07402 a$, respectively. For the optimum $60 / 40$ coupler the radii $r_{1}, \ldots, r_{7}$ are equal to $0.19067 a, 0.20612 a$, $0.27986 a, 0.22087 a, 0.29448 a, 0.05676 a$, and 0.08661a, respectively. In the case of the optimum $3-\mathrm{dB}$ coupler they are equal to $0.18872 a, 0.21608 a, 0.29209 a, 0.22717 a, 0.29884 a$, $0.05676 a$, and $0.08701 a$, respectively.

In the case of the MZI phasing section, the maximum phase error shown in Fig. 7 is within 0.17 rad of the optimal value. In the case of the ring phasing sections shown in Fig. 8, the phase error is within $0.26 \mathrm{rad}$ of the optimum for the upper ring, while for the lower ring phasing section, the phase error is within 0.27 rad of the optimum. The obtained values of $r_{c}, r_{s}$ and $\Delta L$ of the two CROW subsections forming the MZI phasing section are $0.11536 a, 0.12447 a$, and $91.8 \mu \mathrm{m}$, and $0.1163 a, 0.06585 a$, and $81 \mu \mathrm{m}$, respectively. For the upper ring they are $0.10652 a$, $0.13314 a$, and $94.5 \mu \mathrm{m}$, and $0.09636 a, 0.10043 a$, and $86.4 \mu \mathrm{m}$, with a total ring length of $331.2 \mu \mathrm{m}$, while for the lower ring they are $0.10694 a, 0.13202 a$, and $94.5 \mu \mathrm{m}$, and $0.1014 a, 0.10503 a$, and $77.4 \mu \mathrm{m}$, with a total length of $327.6 \mu \mathrm{m}$.

The calculated transmittivity and reflectivity curves are shown in Figs. 9 and 10. Figs. 9(a) and 10(a) show the curves for the upper side of the structure in Fig. 1, having the input port reflectivity and output port 1 transmittivity, while the curves in Figs. 9(b) and 10(b) are for the lower side, showing the reflectivity leakage out of the unused input port and the transmittivity of output port 2. In Fig. 9 the results are calculated taking into account only the coupler losses, and it can be seen that the upper output passband transmittivity varies between $85 \%$ and $93 \%$, with a residual stop-band transmittivity no higher than $8 \%$. The reflectivity on the input port is of $10 \%$ or lower. For the lower output port, passband transmittivity is between $85 \%$ 


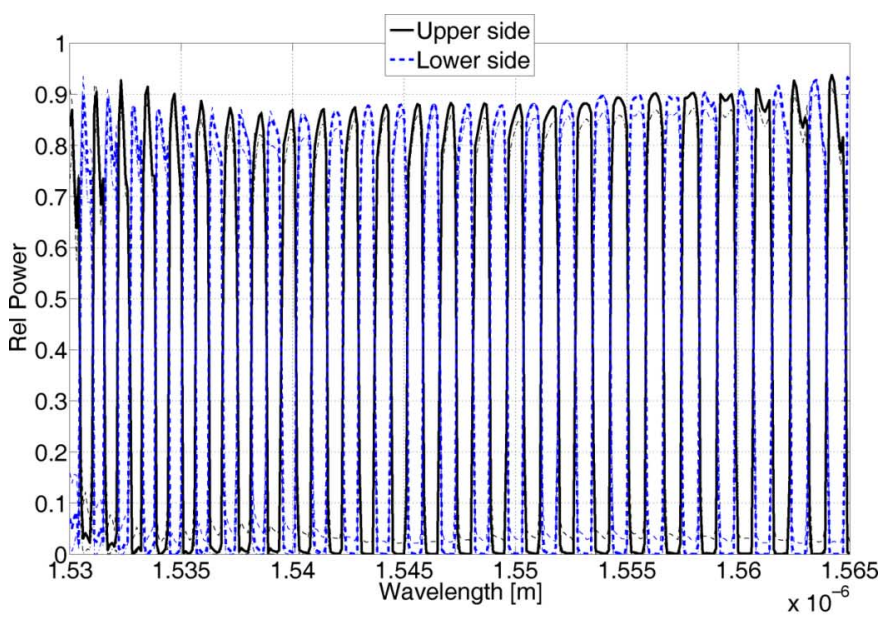

(a)

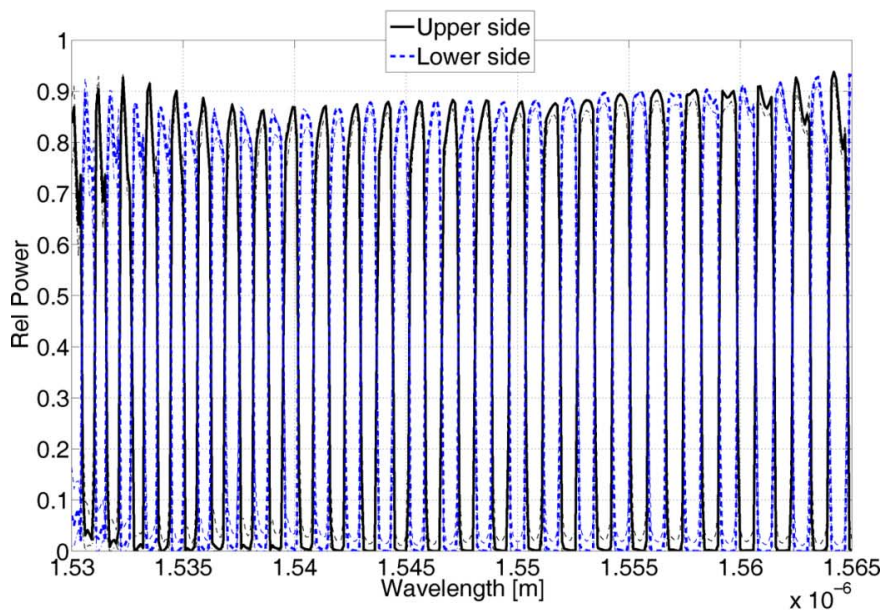

(b)

Fig. 11. Maximum error limits on transmittivity (dashed) compared with errorless case (solid) when a maximum phase error of $0.1 \pi$ is applied to (a) the MZI long arm phase and to (b) the lower ring phase.

and $93 \%$, with a residual no higher than $4 \%$. The reflectivity leakage on the unused port is of $20 \%$ or lower.

The results in Fig. 10 are calculated estimating the 3-D confinement losses of the waveguides on C-band. Preliminary 3-D FEM simulations have shown that a membrane-type air-clad fabrication technique would give ideal-case losses negligible with respect to the building blocks. Available experimental results examine silicon-slab waveguides, so we reviewed similar air-clad waveguides with low losses on C-band. State-of-the-art techniques for membrane-type waveguides give a measured loss as low as $0.6 \mathrm{~dB} / \mathrm{mm}$ [22]. This is numerically fitted in [23] showing a low-loss bandwidth wide enough to cover C-band. It also shows that improvements in the fabrication process can reduce the loss to $0.2 \mathrm{~dB} / \mathrm{mm}$ or lower, in the range of our simulations; however, we considered the result in [22] to be reasonable for a current fabrication process. In Fig. 10 it can be seen that the output passband transmittivity is lowered to values between $71 \%$ and $78 \%$. The residual stop-band transmittivity is no higher than $6 \%$ on the lower side, and almost unaffected on the upper side. Reflectivity on input port and reflectivity leakage on unused port are lowered by $2 \%$ to $4 \%$.

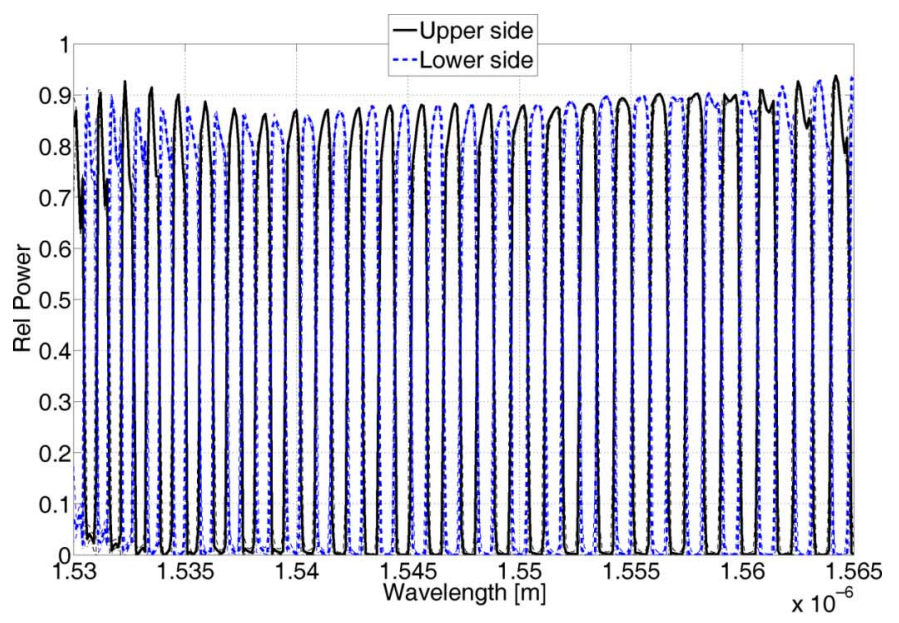

(a)

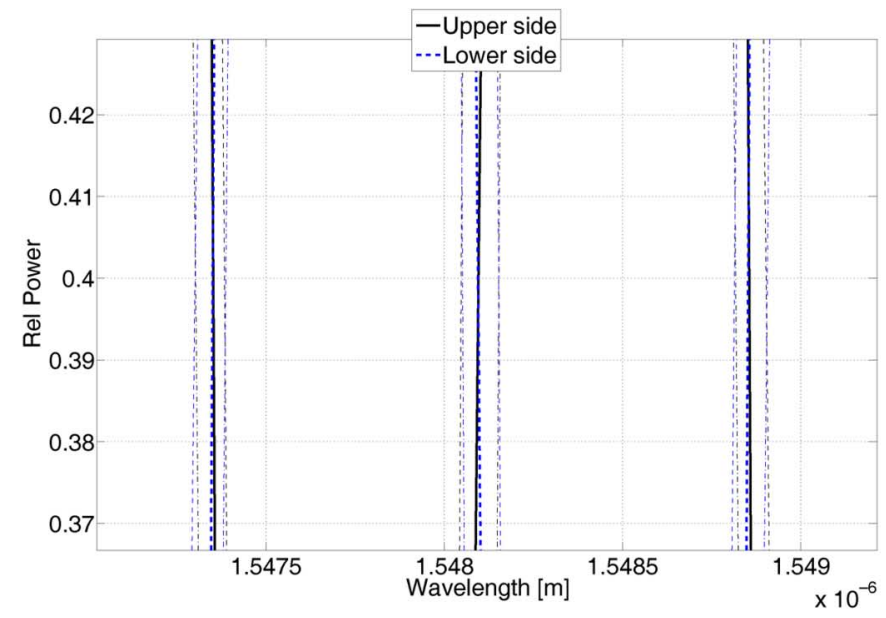

(b)

Fig. 12. (a) Maximum error limits on transmittivity (dashed) compared with errorless case (solid) when a maximum phase error of $0.1 \pi$ is applied to the upper ring phase. (b) Wavelength shift of crossing points.

\section{SEnsitivity Analysis}

A sensitivity analysis has been performed on the model, to evaluate the impact of parameter tolerances in the MZI and ring phasing sections, by introducing a phase error, and in the couplers, by introducing a symmetrical error in the coupling ratios of the through and coupled ports. The errors are evaluated by executing many simulation runs, each with a different random error respecting the maximum value, and taking the limit transmittivity values.

A maximum error of $0.1 \pi$ on the MZI upper arm phase results in a penalty between $2 \%$ and $6 \%$ on the transmittivity values both in passband and stop-band, as shown in Fig. 11(a). A similar effect is observed for the lower arm, as the fundamental parameter in this case is the phase difference between the arms. In Fig. 11(b), the penalty is shown when a maximum error of $0.1 \pi$ is introduced on the lower ring resonator phase, with similar penalty values as the previous case. It can be noticed that in this case, in the central part of the band the effect is less detrimental, especially on the stop-band where the penalty is reduced by about $2 \%$.

An interesting effect is shown by applying the phase variation to the upper ring, which has little effect on the passband and 


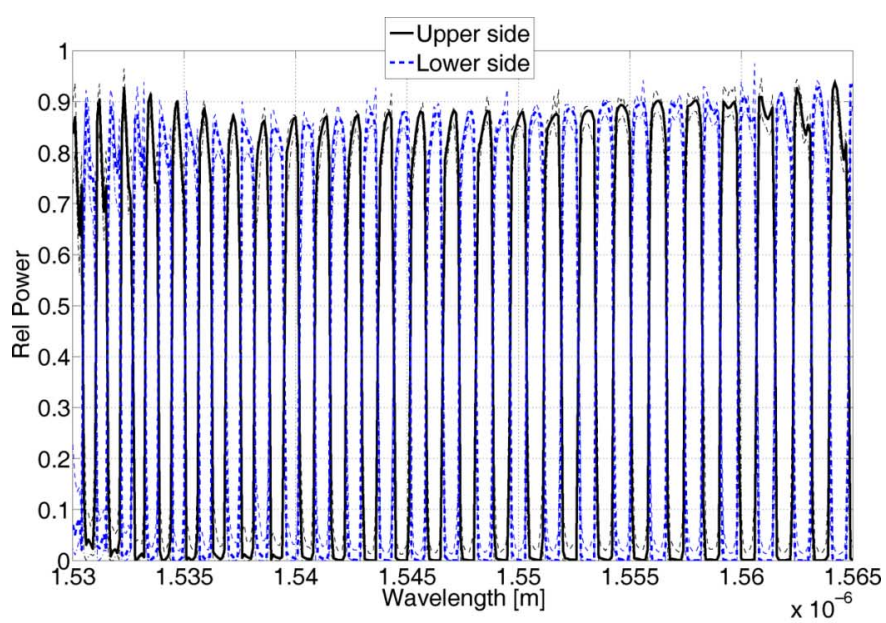

(a)

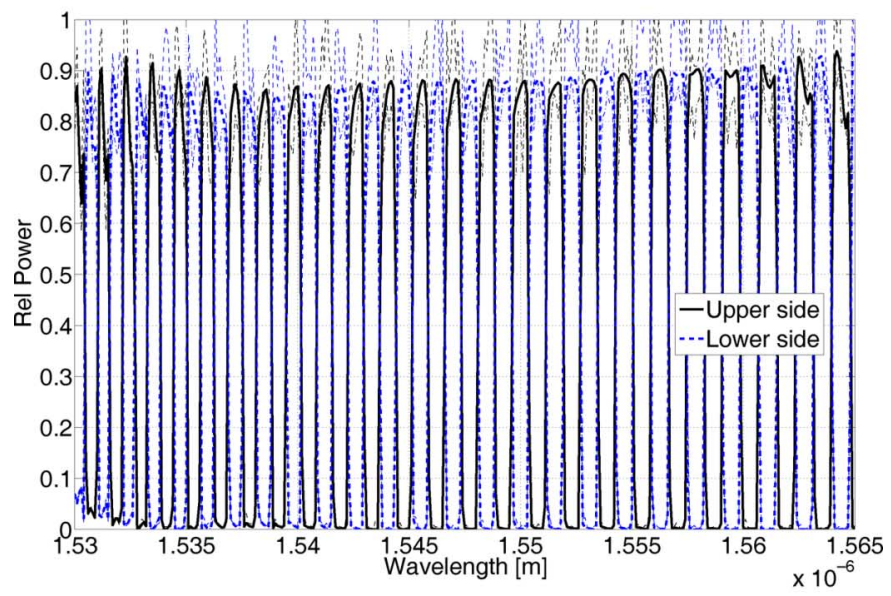

(b)

Fig. 13. Maximum error limits on transmittivity (dashed) compared with errorless case (solid) when a maximum phase error of $1 \%$ is applied to the coupling ratios (a) of the 60/40 coupler and (b) of the 97/03 coupler.

stop-band values as shown in Fig. 12(a), but causes a consistent shift of the entire spectrum by $0.05 \mathrm{~nm}$ on average for a phase variation of $0.1 \pi$, which can be used for a fine tuning of the channel grid, as shown in Fig. 12(b). A greater effect is shown by considering the errors on the coupling ratios in Fig. 13, where the coupling ratios of the 60/40 coupler and of the 97/03 coupler are varied by a $1 \%$ maximum error: for the $60 / 40$ coupler in Fig. 13(a), it produces a maximum change of $5 \%$ in the passband and $4 \%$ in the stop-band, with a more consistent effect on the stop-band. The effect is much greater for the 97/03 coupler in Fig. 13(b), producing wide variations by as much as $-10 \%$ to $+17 \%$ in the passband, while the stop-band effect is negligible.

\section{CONCLUSION}

A C-band PCW interleaver using CROW ring resonators is designed exploiting a GA optimization approach. The filter components are optimized for reduced reflection and high phase linearity, obtaining transmission spectra of $97 \%$ or higher over the whole C-band for PCW-to-CROW tapers and CROW bends. An S-matrix approach is applied to compute the transmittivity and reflectivity of the interleaver, achieving best-case transmission between $85 \%$ and $93 \%$ in the passband and lower than $8 \%$ in the stopband. A sensitivity analysis has been performed to evaluate the effect of phase errors and coupling ratio errors, revealing the critical role of the short-arm coupler and showing a greater sensitivity to coupling errors, varying the passband transmission by as much as $17 \%$.

\section{REFERENCES}

[1] T. P. White, L. C. Botten, R. C. McPhedran, and C. Martijn de Sterke, "Ultracompact resonant filters in photonic crystals," in Opt. Lett., Dec. 15, 2003, vol. 28, pp. 2452-2454.

[2] N. Yokoi, T. Fujisawa, K. Saitoh, and M. Koshiba, "Apodized photonic crystal waveguide gratings," in Opt. Exp., May 15, 2006, vol. 14, pp. 4459-4468.

[3] A. Shinya, S. Mitsugi, E. Kuramochi, and M. Notomi, "Ultrasmall multi-channel resonant-tunneling filter using mode gap of width-tuned photonic-crystal waveguide," in Opt. Exp., May 30, 2005, vol. 13, pp. 4202-4209.

[4] M. Y. Tekeste and J. M. Yarrison-Rice, "High efficiency photonic crystal based wavelength demultiplexer," in Opt. Exp., Aug. 21, 2006, vol. 14, pp. 7931-7942.

[5] M. Qiu, M. Mulot, M. Swillo, S. Anand, B. Jaskorzynska, A. Karisson, M. Kamp, and A. Forchel, "Photonic crystal optical filter based on contra-directional waveguide coupling," in Appl. Phys. Lett., Dec. 22, 2003, vol. 83, pp. 5121-5123.

[6] T. Niemi, L. H. Frandsen, K. K. Hede, A. Harpoth, P. I. Borel, and M. Kristensen, "Wavelength-division demultiplexing using photonic crystal waveguides," IEEE Photon. Technol. Lett., vol. 18, no. 1, pp. 226-228, Jan. 2006.

[7] S. Cao, J. Chen, J. N. Damask, C. R. Doerr, L. Guiziou, G. Harvey, Y. Hibino, H. Li, S. Suzuki, K.-Y. Wu, and P. Xie, "Interleaver technology: Comparisons and application requirements," J. Lightw. Technol., vol. 22, no. 1, pp. 281-289, Jan. 2004.

[8] Y. Akahane, T. Asano, H. Takano, B. S. Song, Y. Takana, and S. Noda, "Two-dimensional photonic-crystal-slab channeldrop filter with flat-top response," in Opt. Exp., Apr. 4, 2005, vol. 13, pp. 2512-2530.

[9] D. Park, S. Kim, I. Park, and H. Lim, "Higher order optical resonant filters based on coupled defect resonators in photonic crystals," J. Lightw. Technol., vol. 23, no. 5, pp. 1923-1928, May 2005.

[10] M. Koshiba, "Wavelength division multiplexing and demultiplexing with photonic crystal waveguide couplers," J. Lightw. Technol., vol. 19, no. 12, pp. 1970-1975, Dec. 2001.

[11] S. Boscolo, M. Midrio, and C. G. Someda, "Coupling and decoupling of electromagnetic waves in parallel 2-D photonic crystal waveguides," IEEE J. Quantum Electron., vol. 38, no. 1, pp. 47-53, Jan. 2002.

[12] Y. Tanaka, H. Nakamura, Y. Sugimoto, N. Ikeda, K. Asakawa, and K. Inoue, "Coupling properties in a 2-D photonic crystal slab directional coupler with a triangular lattice of air holes," IEEE J. Quantum Electron., vol. 41, no. 1, pp. 76-84, Jan. 2005.

[13] M. H. Shih, W. J. Kim, W. Kuang, J. R. Cao, H. Yukawa, S. J. Choi, J. D. O'Brien, P. D. Dapkus, and W. K. Marshall, "Two-dimensional photonic crystal Mach-Zehnder interferometers," Appl. Phys. Lett., vol. 84, pp. 460-462, Jan. 16, 2004.

[14] K. Jinguji and M. Oguma, "Optical half-band filters," J. Lightw. Technol., vol. 18, no. 2, pp. 252-259, Feb. 2000.

[15] A. Martinez, A. Griol, P. Sanchis, and J. Marti, "Mach-Zehnder interferometer employing coupled-resonator optical waveguides," in Opt. Lett., Mar. 15, 2003, vol. 28, pp. 405-407.

[16] A. Yariv, Y. Xu, R. K. Lee, and A. Scherer, "Coupled-resonator optical waveguide: A proposal and analysis," in Opt. Lett., Jun. 1, 1999, vol. 24, pp. 711-713.

[17] F. Cuesta-Soto, B. García-Baños, and J. Martí, "Compensating intermodal dispersion in photonic crystal directional couplers," Opt. Lett., vol. 30, pp. 3156-3158, Dec. 1, 2005.

[18] Y. Tsuji and M. Koshiba, "Finite element method using port truncation by perfectly matched layer boundary conditions for optical waveguide discontinuity problem," J. Lightw. Technol., vol. 20, no. 3, pp. 463-468, Mar. 2002.

[19] J. Goh, I. Fushman, D. Englund, and J. Vučković, "Genetic optimization of photonic bandgap structures," Opt. Exp., vol. 15, pp. 8218-8230, Jun. 18, 2007.

[20] N. Kono, N. Yokoi, K. Saitoh, and M. Koshiba, "Proposal for miniaturized interleaver with flat-top passbands utilizing coupled-resonator optical waveguide rings iwww.www.n photonic crystals," in Proc. OFC 2007, Anaheim, CA, Mar. 26-29, 2007, JWA22. 
[21] K. Jinguji, "Synthesis of coherent two-port optical delay-line circuit with ring waveguides," J. Lightw. Technol., vol. 14, no. 8, pp. 1882-1898, Aug. 1996.

[22] M. Notomi, A. Shinya, S. Mitsugi, E. Kuramochi, and H.-Y. Ryu, "Waveguides, resonators and their coupled elements in photonic crystal slabs," Opt. Exp., vol. 12, pp. 1551-1561, Apr. 19, 2004.

[23] D. Gerace and L. C. Andreani, "Low-loss guided modes in photonic crystal waveguides," Opt. Exp., vol. 13, pp. 4939-4951, Jun. 27, 2005.

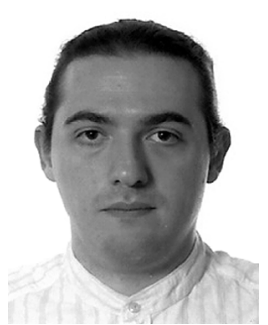

Lorenzo Rosa (S'97-M'02-S'05-M'08) was born in Parma, Italy, in 1974. He received the Laurea degree in electronics engineering in 2003, and the Ph.D. degree in information technology in 2007, both from the University of Parma, Parma, Italy.

Since October 2007, he has been working as a GCOE Postdoctoral Fellow at the Division of Media and Network Technologies, Graduate School of Information Science and Technology, Hokkaido University, Sapporo, Hokkaido, Japan. His research interests include photonic-crystal fibers and waveguides, integrated optics components, finite-element methods for electromagnetics, and antenna design.

Dr. Rosa is a member of the Optical Society of America (OSA).

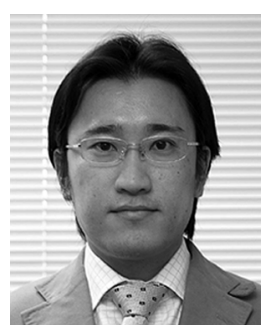

Kunimasa Saitoh (S'00-M'01) was born in Hokkaido, Japan. He received the B.S., M.S., and $\mathrm{Ph} . \mathrm{D}$. degrees in electronic engineering from Hokkaido University, Sapporo, Japan, in 1997, 1999, and 2001, respectively.

From 1999 to 2001, he was a Research Fellow of the Japan Society for the Promotion of Science. From 2001 to 2005, he was a Research Associate of Graduate School of Engineering at Hokkaido University. In 2005, he became an Associate Professor at Graduate School of Information Science and Technology, Hokkaido University. He has been engaged in research on fiber optics, nanophotonics, integrated optical devices, and computer-aided design and modeling of guided-wave devices using finite-element method, beam propagation method, and so on. He is an author or coauthor of more than 90 research papers in refereed international journals.

Prof. Saitoh is a member of the Institute of Electronics, Information and Communication Engineers (IEICE) and the Optical Society of America (OSA). In 1999 and 2002, he was awarded the Excellent Paper Award and the Young Scientist Award from the IEICE, respectively, and in 2008, the Young Scientists' Prize of the Commendation for Science and Technology from the Ministry of Education, Culture, Sports, Science, and Technology (MEXT), Government of Japan
Kuniaki Kakihara was born in Furano, Japan, on January 7, 1983. He received the B.S. and M.S. degrees in electronic engineering from Graduate School of Information Science and Technology, Hokkaido University, Sapporo, Japan, in 2006 and 2008, respectively. He is currently working toward the Ph.D. degree at the Graduate School of Information Science and Technology, Hokkaido University.

He has been engaged in research on wave electronics, integrated optical devices, and computer-aided design and modeling of guided-wave devices using the finite-element method and beam propagation method.

Mr. Kakihara is a student member of the Institute of Electronics, Information and Communication Engineers (IEICE) of Japan.

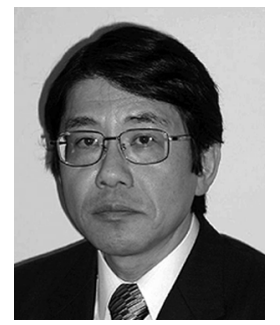

Masanori Koshiba (M'76-SM'87-F'03) was born in Sapporo, Japan. He received the B.S., M.S., and $\mathrm{Ph} . \mathrm{D}$. degrees in electronic engineering from Hokkaido University, Sapporo, Japan, in 1971, 1973, and 1976, respectively.

In 1976, he joined the Department of Electronic Engineering, Kitami Institute of Technology, Kitami, Japan. From 1979 to 1987, he was an Associate Professor of Electronic Engineering at Hokkaido University, and in 1987, he became a Professor there. He has been engaged in research on wave electronics, including microwaves, millimeter-waves, lightwaves, surface acoustic waves (SAW), magnetostatic waves (MSW), and electron waves, and computer-aided design and modeling of guided-wave devices using finite-element method, boundary-element method, beam propagation method, and so on. He is an author or coauthor of more than 320 research papers in English and of more than 130 research papers in Japanese both in refereed journals. He is an author of the books Optical Waveguide Analysis (McGraw-Hill, 1992) and Optical Waveguide Theory by the Finite Element Method (Kluwer, 1992), and is a coauthor of the books Analysis Methods for Electromagnetic Wave Problems (Artech House, 1990), Analysis Methods for Electromagnetic Wave Problems, Vol. Two (Artech House, 1996), Ultrafast and Ultra-parallel Optoelectronics (Wiley, 1995), and Finite Element Software for Microwave Engineering (New York: Wiley, 1996).

Prof. Koshiba is a fellow of the Institute of Electronics, Information, and Communication Engineers (IEICE), and is a member of the Institute of Electrical Engineers of Japan and the Institute of Image Information and Television Engineers of Japan. In 1987, 1997, and 1999, he was awarded the Excellent Paper Awards from the IEICE, in 1998, the Electronics Award from the IEICE-Electronics Society, and in 2004, the Achievement Award from the IEICE. From 1999 to 2000, he served as a President of the IEICE-Electronics Society, and in 2002, he served as a Chair of the IEEE Lasers and Electro-Optics Society (LEOS) Japan Chapter. Since 2003, he has served on the Board of Directors of the IEICE. 